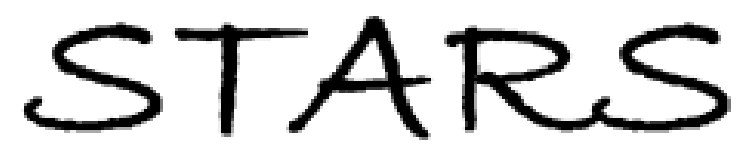

University of Central Florida

STARS

$1-1-2013$

\title{
Driven translocation of a semi-flexible chain through a nanopore: A Brownian dynamics simulation study in two dimensions
}

Ramesh Adhikari

University of Central Florida

Aniket Bhattacharya

University of Central Florida

Find similar works at: https://stars.library.ucf.edu/facultybib2010

University of Central Florida Libraries http://library.ucf.edu

This Article is brought to you for free and open access by the Faculty Bibliography at STARS. It has been accepted for inclusion in Faculty Bibliography 2010 s by an authorized administrator of STARS. For more information, please contactSTARS@ucf.edu.

\section{Recommended Citation}

Adhikari, Ramesh and Bhattacharya, Aniket, "Driven translocation of a semi-flexible chain through a nanopore: A Brownian dynamics simulation study in two dimensions" (2013). Faculty Bibliography 2010 s. 3589.

https://stars.library.ucf.edu/facultybib2010/3589

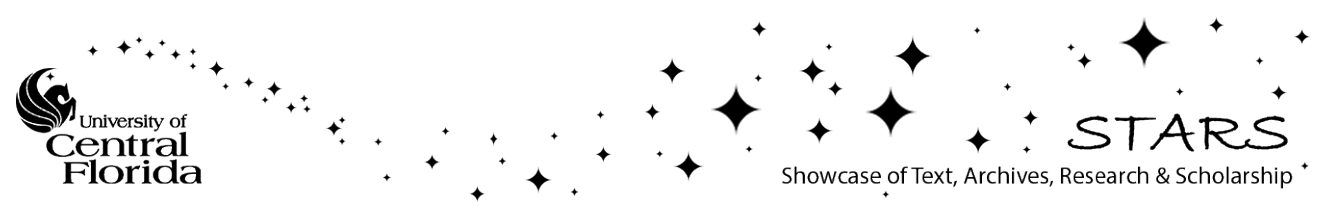




\section{Driven translocation of a semi-flexible chain through a nanopore: A Brownian dynamics simulation study in two dimensions}

Cite as: J. Chem. Phys. 138, 204909 (2013); https://doi.org/10.1063/1.4807002

Submitted: 12 February 2013 . Accepted: 03 May 2013. Published Online: 30 May 2013

Ramesh Adhikari, and Aniket Bhattacharya

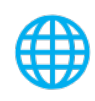

View Online
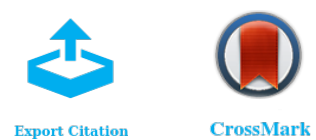

Export Citation

\section{ARTICLES YOU MAY BE INTERESTED IN}

Translocation is a nonequilibrium process at all stages: Simulating the capture and translocation of a polymer by a nanopore

The Journal of Chemical Physics 145, 154902 (2016); https://doi.org/10.1063/1.4964630

Role of non-equilibrium conformations on driven polymer translocation

The Journal of Chemical Physics 148, 024903 (2018); https://doi.org/10.1063/1.4994204

Polymer translocation through a hole

The Journal of Chemical Physics 111, 10371 (1999); https://doi.org/10.1063/1.480386

Where in the world is AIP Publishing? Find out where we are exhibiting next 


\title{
Driven translocation of a semi-flexible chain through a nanopore: A Brownian dynamics simulation study in two dimensions
}

\author{
Ramesh Adhikari and Aniket Bhattacharya ${ }^{\text {a) }}$ \\ Department of Physics, University of Central Florida, Orlando, Florida 32816-2385, USA
}

(Received 12 February 2013; accepted 3 May 2013; published online 30 May 2013)

\begin{abstract}
We study translocation dynamics of a semi-flexible polymer chain through a nanoscopic pore in two dimensions using Langevin dynamics simulation in presence of an external bias $F$ inside the pore. For chain length $N$ and stiffness parameter $\kappa_{b}$ considered in this paper, we observe that the mean first passage time $\langle\tau\rangle$ increases as $\left\langle\tau\left(\kappa_{b}\right)\right\rangle \sim\left\langle\tau\left(\kappa_{b}=0\right)\right\rangle l_{p}^{a_{N}}$, where $\kappa_{b}$ and $l_{p}$ are the stiffness parameter and persistence length, respectively, and $a_{N}$ is a constant that has a weak $N$ dependence. We monitor the time dependence of the last monomer $x_{N}(t)$ at the cis compartment and calculate the tension propagation time (TP) $t_{t p}$ directly from simulation data for $\left\langle x_{N}(t)\right\rangle \sim t$ as alluded in recent nonequlibrium TP theory [T. Sakaue, Phys. Rev. E 76, 021803 (2007)] and its modifications to Brownian dynamics tension propagation theory [T. Ikonen, A. Bhattacharya, T. Ala-Nissila, and W. Sung, Phys. Rev. E 85, 051803 (2012); J. Chem. Phys. 137, 085101 (2012)] originally developed to study translocation of a fully flexible chain. We also measure $t_{t p}$ from peak position of the waiting time distribution $W(s)$ of the translocation coordinate $s$ (i.e., the monomer inside the pore), and explicitly demonstrate the underlying TP picture along the chain backbone of a translocating chain to be valid for semi-flexible chains as well. From the simulation data, we determine the dependence of $t_{t p}$ on chain persistence length $l_{p}$ and show that the ratio $t_{t p} /\langle\tau\rangle$ is independent of the bias $F$. (C) 2013 AIP Publishing LLC. [http://dx.doi.org/10.1063/1.4807002]
\end{abstract}

\section{INTRODUCTION}

The phenomenon of polymer translocation $(\mathrm{PT})^{1,2}$ through narrow channels and pores continues to be an active field of research for more than a decade. PT is of particular interest in the context of biopolymers as translocation is an important ubiquitous process in molecular biology. Translocation of DNA and RNA across nuclear pores, protein transport through membrane channels, and virus injection are examples of such processes. ${ }^{3}$ Naturally, a microscopic and fundamental understanding of PT processes in living organisms is essential in health related issues. Understanding PT is also immensely important for making fast, efficient, and low cost single molecule analyses devices. In a series of pioneering experiments using single stranded as well as double stranded DNA translocating through $\alpha$-hemolysin protein pore and synthetic nanopores, ${ }^{4-7}$ where the histogram of the mean first passage time (MFPT) was obtained by measuring the fluctuation in the channel current, it was demonstrated that a nanopore can be used to determine sequences of a heteropolymer. Recently "nano-pore" based techniques have been commercialized and are being used to detect sequences. ${ }^{8}$ Significant advancement has been made to fabricate uniformly sized nanopores and nanopore arrays for fast parallel high throughput DNA analysis. ${ }^{9,10}$

These exciting experiments have provided enough enthusiasm to develop a proper theoretical framework for polymer translocation through a nanopore. Sung and Park ${ }^{11}$ and

\footnotetext{
a)aniket@physics.ucf.edu
}

Muthukumar ${ }^{12}$ considered translocation as a one-dimensional barrier crossing problem and derived expression for the translocation exponent $\alpha\left(\langle\tau\rangle \sim N^{\alpha}\right)$ using a free energy expression for a polymer threaded through the pore (Fig. 1). These initial predictions were followed by many others ${ }^{13-23}$ using back of the envelope estimates and dynamical scaling arguments, ${ }^{14,15}$ analyzing folds of the chains, ${ }^{16}$ incorporation of memory effects, ${ }^{17-19}$ mass and energy conservations, ${ }^{20,21}$ and tension propagation(TP) along the chain backbone. ${ }^{20}$ The TP theory captures the true nonequlibrium aspects for the case of driven translocation. The original theory, which was introduced for an infinite chain has been further refined by Ikonen et al. ${ }^{22-24}$ and Dubbeldam et al. ${ }^{25}$ to study the TP in a finite chain. Ikonen et al. ${ }^{22-24}$ extended the TP theory to a Brownian dynamics scheme (Brownian dynamics tension propagation (BDTP)) and emphasized the role of non-negligible pore friction for finite chains which introduces correction to scaling in the translocation exponent. ${ }^{24}$ BDTP theory explains scattered values of the translocation exponent $\alpha$ and provides a unifying picture of driven translocation using the TP idea. These experimental and theoretical developments have been supplemented by a large number of simulation studies which played crucial role in the theoretical developments in the field. ${ }^{22-45}$ Along with simulation studies of coarse-grained model $a b$ initio and atomic resolution Brownian dynamics have been carried out to predict the DNA sequence dependence on ion current. ${ }^{46}$

Almost all of the aforementioned theoretical and simulation studies have been addressed in the context of a fully flexible chain. ${ }^{4-49}$ However, in order to capture some realistic aspects of a translocating of a DNA through a nanopore, 


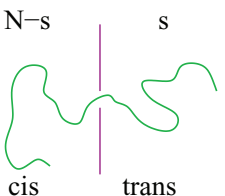

FIG. 1. Minimalist view of a polymer chain translocating through an ideal pore in a thin wall from cis to trans side in terms of the translocation $(s)$ coordinate. The picture shows an instant of translocation when $s$ segments are at the trans side with remaining $N-s$ segments at the cis side of a chain of length $N$.

one needs to consider the chain stiffness. ${ }^{47-49}$ The purpose of this paper is twofold. First to extend the simulation studies of polymer translocation for a semi-flexible chain. Second, use the simulation results to validate some aspects of the tension propagation phenomenon which has been very useful to explain apparent discrepancies of the simulation studies for finite chains. We find that many aspects of the TP picture, which has been developed for a fully flexible chain remain valid for semi-flexible chains as well. By monitoring the dynamics of the monomers as a function of the chain length, chain stiffness, and the driving force, we have been able to obtain a complete picture of the translocation process. One of the salient aspects of this study is that our simulation results provide direct demonstration of the TP along the chain backbone and by comparing the tension propagation time $t_{t p}$ obtained (i) from the time dependence of the last monomer and (ii) from the peak position of the residence time $W(s)$ of the translocation $(s)$ coordinate our studies validate the theoretical prediction of time dependent drag on the translocating chain.

\section{THE MODEL}

We have used a bead spring model of a polymer chain with excluded volume, spring, and bending potentials as follows. ${ }^{50}$ The excluded volume interaction between any two monomers is given by short range Lennard-Jones (LJ) potential

$$
\begin{aligned}
U_{\mathrm{LJ}}(r) & =4 \epsilon\left[\left(\frac{\sigma}{r}\right)^{12}-\left(\frac{\sigma}{r}\right)^{6}\right]+\epsilon \text { for } r \leq 2^{1 / 6} \sigma \\
& =0 \text { for } r>2^{1 / 6} \sigma .
\end{aligned}
$$

Here, $\sigma$ is the effective diameter of a monomer, and $\epsilon$ is the strength of the potential. The connectivity between neighboring monomers is modeled as a Finite Extension Nonlinear Elastic (FENE) spring with

$$
U_{\mathrm{FENE}}(r)=-\frac{1}{2} k R_{0}^{2} \ln \left(1-r^{2} / R_{0}^{2}\right),
$$

where $r$ is the distance between consecutive monomers, $k$ is the spring constant and $R_{0}$ is the maximum allowed separation between connected monomers. ${ }^{50}$ The chain stiffness is introduced by adding an angle dependent interaction between successive bonds as (Fig. 2):

$$
U_{\text {bend }}\left(\theta_{i}\right)=\kappa_{b}\left(1-\cos \theta_{i}\right) \text {. }
$$

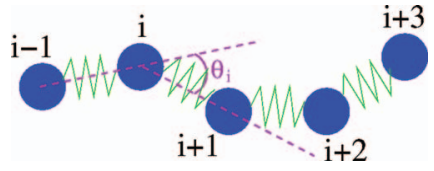

FIG. 2. Bead-spring model of a polymer chain with bending angle $\theta_{i}$ subtended by the vectors $\vec{b}_{i}=\vec{r}_{i}-\vec{r}_{i-1}$ and $\vec{b}_{i+1}=\vec{r}_{i+1}-\vec{r}_{i}$.

Here $\theta_{i}$ is the angle between the bond vectors $\vec{b}_{i-1}=\vec{r}_{i}-\vec{r}_{i-1}$ and $\vec{b}_{i}=\vec{r}_{i+1}-\vec{r}_{i}$, respectively, as shown in Fig. 2. The strength of the interaction is characterized by the bending rigidity $\kappa_{b}$.

The purely repulsive wall consists of one monolayer(line) of immobile LJ particles of diameter $\sigma$ along the $y$ axis at $x=0$. The pore is created by removing two particles at the center (Fig. 3). Inside the pore, the polymer beads experience a constant force $F$ and a repulsive potential from the inside wall of the pore. We use the Langevin dynamics with the following equation of motion for the $i$ th monomer:

$$
m \ddot{\vec{r}}_{i}=-\nabla\left(U_{\mathrm{LJ}}+U_{\mathrm{FENE}}+U_{\mathrm{bend}}+U_{\mathrm{ext}}\right)-\zeta \vec{v}_{i}+\vec{\eta}_{i} .
$$

Here $\zeta$ is the monomer friction coefficient and $\vec{\eta}_{i}(t)$, is a Gaussian white noise with zero mean at a temperature $\mathrm{T}$, and satisfies the fluctuation-dissipation relation:

$$
\left\langle\vec{\eta}_{i}(t) \cdot \vec{\eta}_{j}\left(t^{\prime}\right)\right\rangle=6 k_{B} T \zeta \delta_{i j} \delta\left(t-t^{\prime}\right) .
$$

The reduced units of length, time, and temperature are chosen to be $\sigma, \sigma \sqrt{\frac{m}{\epsilon}}$, and $\epsilon / k_{B}$, respectively. For the spring potential, we have chosen $k=30$ and $R_{0}=1.5 \sigma$, the friction coefficient $\eta=0.7$, the temperature is kept at $1.2 / k_{B}$, and $U_{\text {ext }}=-F x$ is an external potential which produces a constant force $F$ inside the pore. The value of this external bias is kept at $F \sigma / \epsilon$ $=5.0$ throughout the simulation. The choice of the FENE potential along with the $\mathrm{LJ}$ interaction parameters ensures that the average bond-length in the bulk $\left\langle b_{l}\right\rangle=0.971$. With the choice of these parameters probability of chain crossing is very low. These parameters have been chosen to be the same as in our recent studies ${ }^{22,23,27,28}$ of polymer translocation of

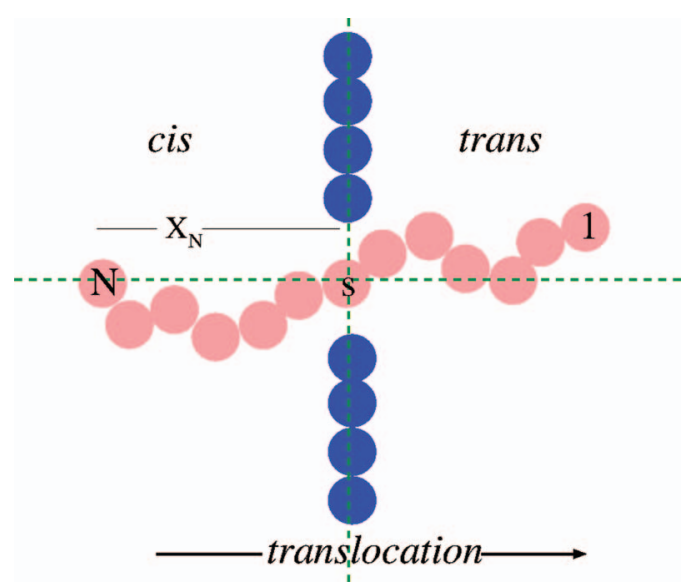

FIG. 3. Representation of the $s$ coordinate (sth) monomer inside the pore in the bead-spring model of a translocating chain used in our simulation. The figure shows a $N=13$ chain having the 7 th monomer $(s=7)$ inside the pore and the remaining $N-s=6$ monomers at the cis side. The springs joining the monomers are not shown in Fig. 3. Translocation occurs from the cis to the trans side. 
flexible chains for ready comparison of results. We also find that the average bond-length $\left\langle b_{l}\right\rangle$ is almost independent of the range of chain stiffness parameter $\left(\kappa_{b}=0-32\right)$ used in our simulation. The equation of motion is integrated with the reduced time step $\Delta t=0.01$ following the algorithm proposed by van Gunsteren and Berendsen. ${ }^{54}$

\section{SIMULATION RESULTS}

We have studied 5 different chain lengths $N=16,32,64$, 128 , and 256 for several different values of the bending constant $\kappa_{b}=0-32$. However, for clarity we present only a limited set of data. First we equilibrated the polymer chain by placing the first monomer at the center of the pore. We then allow the polymer to translocate driven by the bias present uniformly inside the pore. For the translocation related properties, we have taken statistics from at least 2000 to 5000 independent runs, as needed to obtain good statistics.

\section{A. Mean first passage time $\langle\tau\rangle$ and its distribution}

We first studied how the MFPT varies with the chain stiffness leaving everything else the same. We find that the MFPT monotonically increases with the chain stiffness as shown in Fig. 4(a). Here we provide a plausible physical argument for this increase in MFPT combining TP idea with the Kantor and Kardar estimate of MFPT ${ }^{15}$ which is given by

$$
\langle\tau\rangle \sim\left\langle R_{g}\right\rangle /\left\langle v_{C M}\right\rangle,
$$

where $\left\langle R_{G}\right\rangle$ and $\left\langle v_{C M}\right\rangle$ correspond to the average root mean square radius of gyration and the average velocity of the center of mass of the chain, respectively. According to Eq. (6), the MFPT $\langle\tau\rangle$ will increase provided $\left\langle R_{g}\right\rangle$ increases and $\left\langle v_{C M}\right\rangle$ either decreases, or stays constant. In Figs. 4(b) and 4(c), we show how these two quantities vary as the stiffness is increased. One expects $\left\langle R_{g}\right\rangle$ (and therefore, the average root mean square end-to-end distance $\left\langle R_{N}\right\rangle$ ) to increase for a stiffer chain which is exactly the case. We also observe that $\left\langle v_{C M}\right\rangle$ decreases as a function of the chain stiffness. The decrease in $\left\langle v_{C M}\right\rangle$ for a stiffer chain can be explained using TP idea which

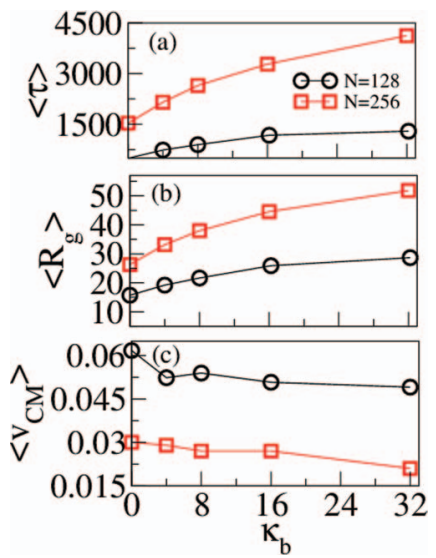

FIG. 4. Variation of (a) MFPT $\langle\tau\rangle$, (b) $\left\langle R_{g}\right\rangle$, and $\left\langle v_{C M}\right\rangle$ as a function of chain stiffness parameter $\kappa_{b}$ for $N=128$ (black circles) and for $N=256$ (red squares), respectively. The lines through points are merely for guide to the eye.

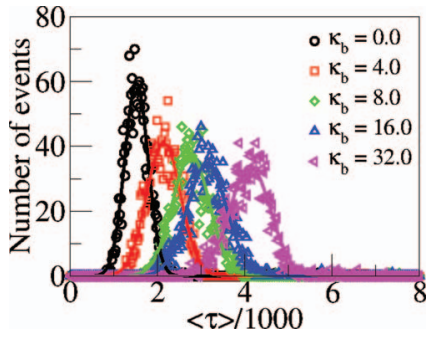

FIG. 5. Histogram of the MFPT for various values of the bending rigidity $\kappa_{b}=0.0$ (black circles), 4.0 (red squares), 8.0 (green diamonds), 16.0 (blue up-triangles), 32.0 (magenta left-triangles) for chain length $N=256$. Each histogram is drawn from 5000 independent runs. Solid lines represent the Gaussian fits to the corresponding data.

is discussed in detail in Sec. III D. In short, this happens due to increase in the relative fraction of monomers at an earlier time on the cis side responding to the bias at the pore which increases the viscous drag on the chain at the cis side. ${ }^{22-24} \mathrm{In}$ Sec. III D, we will resume this discussion and show how the average velocity of the individual monomers inside the pore decreases for a stiffer chain.

We further observe by monitoring the time dependence of the $s$-coordinate (not shown here) that for a stiffer chain a given monomer oscillates back and forth between the cis and trans side more often before making a final exit to the trans side. This is reflected in histogram of the MFPT shown in Fig. 5 which becomes broader with the peak position being shifted at a higher value. This can be understood by noting that compared to a fully flexible chain, the entropic barrier term is reduced by the corresponding chain persistence length $l_{p}$ for a stiffer chain. For a fully flexible chain of length $N$ with $n$ segments at the cis side the entropic barrier is given by

$$
S(N, n) / k_{B}=n \ln n+(N-n) \ln (N-n),
$$

which implies that the change in entropy for $n \rightarrow n+\Delta n$ is $\Delta S=k_{B} \Delta n \ln \left(\frac{n}{N-n}\right)$, where $\Delta n$ represents the change in the number of monomer due to translocation from cis to the trans side. For simplicity if we consider this around $n \sim N / 2$, then the corresponding change in free energy $\Delta F=k_{B} T \Delta n$. Therefore, for $\Delta n= \pm 1$ corresponds to $\Delta F= \pm k_{B} T$. This energy corresponds to roughly $20 \%$ of the energy due to the driving force. Now when the chain becomes stiffer $\Delta n$ $\rightarrow \Delta n / l_{p}$ and this free energy decreases and the entropic penalty for moving back and forth becomes less which increases the MFPT.

We have found (not shown here) that the persistence length $l_{p}$ for the range of $\kappa_{b}$ used here satisfies the relation $l_{P}=2 \frac{\kappa_{b}}{k_{k} T}=-\frac{1}{\ln \langle\cos \theta\rangle}$, where $\theta$ is the equilibrium bond angle (see Fig. 2) which holds strictly for a worm like chain (WLC) ${ }^{51}$ The extension of Flory theory for a semi-flexible chain has been done by Schaefer, Joanny, and Pincus ${ }^{52}$ and by Nakanishi ${ }^{53}$ which states that the end-to-end distance exhibits the following scaling relation:

$$
\left\langle R_{N}\right\rangle \sim N^{\frac{3}{d+2}} l_{p}^{\frac{1}{d+2}},
$$

where $d$ is the physical dimension. For $d=2$, this reduces to $\left\langle R_{N}\right\rangle \sim N^{0.75} l_{p}^{0.25}$. We observe excellent data collapse for the renormalized end-to-end distances $\left\langle R_{N}\right\rangle / l_{p}^{0.25}$ (using 

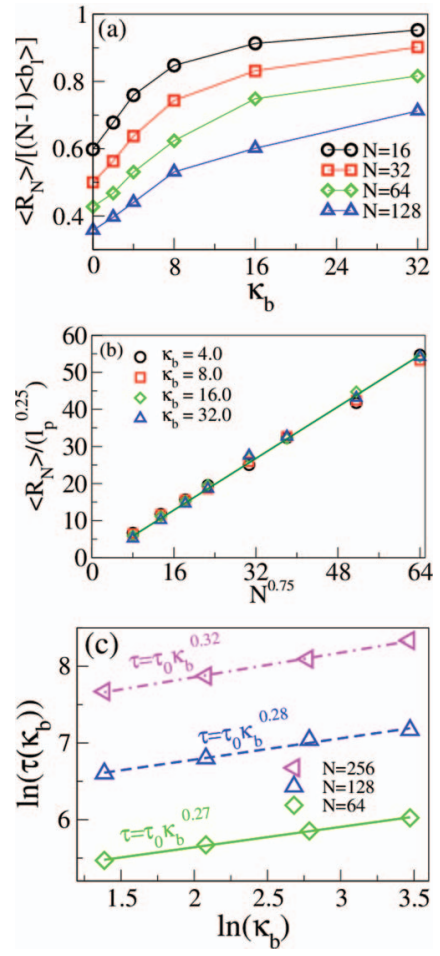

FIG. 6. (a) $\sqrt{\left\langle R_{N}^{2}\right\rangle} /\left((N-1)\left\langle b_{l}\right\rangle\right)$ as a function of $\kappa_{b}$ for different chain length $N=16$ (black circles), 32 (red squares), 64 (green diamonds), and 128 (blue up-triangles), respectively. For a given value of $\kappa_{b}$, the smallest chain are elongated most. (b) Plot of rescaled end-to-end distance $\sqrt{\left\langle R_{N}^{2}\right\rangle} / l_{p}^{0.25}$ ver$\operatorname{sus} N^{0.75}$, where the rescaled end-to-end distances for different chain stiffness collapse on to the same master plot. The solid line is a fit to a straight line. (c) Variation of MFPT $\langle\tau\rangle$ as a function of chain stiffness $\kappa_{b}$ for different chain of length $N=64$ (green diamonds), 128 (blue up-triangles), 256 (magenta left-triangles), respectively, on a log-log scale. The straight line through the points satisfies a simple power law fit.

$l_{P}=2 \frac{\kappa_{b}}{k_{B} T}$ ) for different values of $\kappa_{b}$ shown in Fig. 6(b) as expected from Eq. (8). Since the variation of the velocity of the center of mass is small compared to the variation of chain extension (Fig. 4(c)) as a function of $\kappa_{b}$, this is reflected in the $\log -\log$ plot of $\langle\tau\rangle$ as a function of $\kappa_{b}$ which satisfies a simple power law (Fig. 6(c)). This can be explained as follows. Using Eqs. (6) and (8) in two dimensions (2D), we can write

$$
\begin{aligned}
\left\langle\tau\left(\kappa_{b}\right)\right\rangle & =\frac{\left\langle R_{g}\left(\kappa_{b}=0\right)\right\rangle l_{p}^{0.25}}{\left\langle v_{C M}\left(\kappa_{b}\right)\right\rangle} \\
& =\frac{\left\langle R_{g}\left(\kappa_{b}=0\right)\right\rangle}{\left\langle v_{C M}\left(\kappa_{b}=0\right)\right\rangle} l_{p}^{0.25+\delta},
\end{aligned}
$$

or

$$
\left\langle\tau\left(\kappa_{b}\right)\right\rangle=\left\langle\tau\left(\kappa_{b}=0\right)\right\rangle l_{p}^{0.25+\delta},
$$

where the weak dependence of $\left\langle v_{C M}\left(\kappa_{b}\right)\right\rangle$ on chain persistence length (and possible weak chain length dependence) is accommodated in $\delta$. Since $\kappa_{b}=2 l_{p} / k_{B} T$, therefore a log-log plot of Eq. (9) exhibits a slope $0.25+\delta$ as in Fig. 6(c).

\section{B. Dynamics of the last monomer}

In order to study the effect of the chain stiffness on translocation and to relate our results with the recent nonequilibrium TP theories, ${ }^{20,22,23}$ we have monitored the dy-

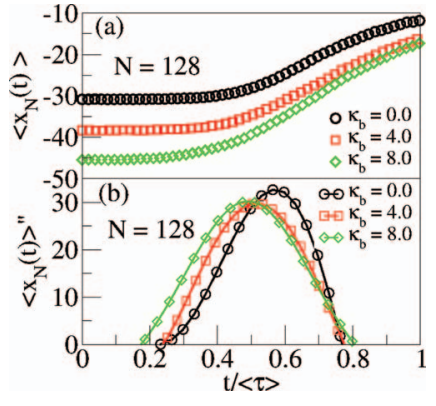

FIG. 7. (a) Plot of $\left\langle x_{N}(t)\right\rangle$ as a function of time scaled by the average translocation time for the chain-length $N=128$ and bending constants $\kappa_{b}=0.0,4.0$, and 8.0, respectively; (b) The corresponding second derivatives $d^{2}\left\langle x_{N}(t)\right\rangle / d t^{2}$ $=\left\langle x_{N}(t)\right\rangle^{\prime \prime}$ (where the peaks correspond to the tension propagation time $t_{t p}$ ). The symbols have the same meaning as in Fig 5.

namics of the last monomer. Recall that in case of a driven translocation, the first monomer of the chain is initially inside the pore at time $t=0$. As it is allowed to move driven by the bias inside the pore, a disturbance (TP) starts propagating at the cis side. One can associate a characteristic time when this disturbance reaches the last monomer. This is called the TP time $t_{t p}$. We will see that along with the MFPT $\langle\tau\rangle$ and its histogram several aspects of polymer translocation can be explained using $t_{t p}$.

A direct way to calculate $t_{t p}$ is to monitor the motion of the last monomer. In Fig. 7(a), we have shown $\left\langle x_{N}(t)\right\rangle$ as a function of $t /\langle\tau\rangle$ where $x_{N}$ is the perpendicular distance of the $N$ th monomer from the wall (see Fig. 3). As expected, the average location of $\left\langle x_{N}(t)\right\rangle$ stays more or less constant until $t \approx t_{t p}$ and then starts decreasing when the last monomer starts moving towards the pore. This time delay to respond to the driving force can be used to define the tension propagation time $t_{t p}$. We have determined $t_{t p}$ from the peak position of the 2nd derivative of $\left\langle x_{N}(t)\right\rangle$ as shown in Fig. 7(b) for several values of the bending constant $\kappa_{b}$. By repeating this exercise, we have determined the $t_{t p}$ directly from the time dependence of the last monomer. However, to calculate $t_{t p}$ from $\left\langle x_{N}(t)\right\rangle$ requires much more statistics than what is needed to determine $\langle\tau\rangle$. We can also determine $t_{t p}$ from the residence time of the individual monomers using the ideas of the tension propagation theory, as discussed in Secs. III C and III D at albeit less computational cost. We have checked that the $t_{t p}$ calculated by these two methods agrees very well providing direct validation of the tension propagation picture of polymer translocation through nanopore. ${ }^{22,23}$

\section{Waiting time distribution}

The waiting time distribution $W(s)$ is defined as the amount of time a monomer $s$ spends inside the pore so that

$$
\sum_{s=1}^{N}\langle W(s)\rangle=\langle\tau\rangle .
$$

Evidently a plot of $W(s)$ as a function of $s$ reveals detailed information about the translocation process of the individual monomers. This quantity has been studied in detail in the past 

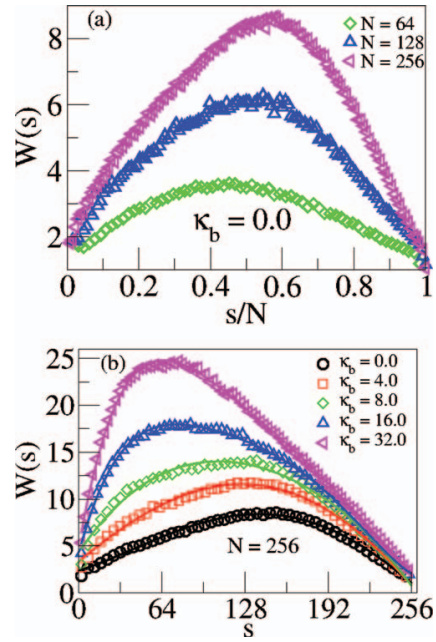

FIG. 8. (a) Residence time of the individual monomers as a function of the reduced coordinate $s / N$ for three chain lengths $N=64,128$, and 256 for $\kappa_{b}$ $=0.0$. (b) Residence time of the individual monomers as a function of $\kappa_{b}$ for chain length $N=256$. Please note that the peak position shifts at a lower $s$-value for a higher value of $\kappa_{b}$.

for fully flexible chains and more recently for semi-flexible chains. Typical plots of $W(s)$ as a function of $s$ are shown in Figs. 8 and 9 where each plot is characterized by a peak $W_{\max }$. The position of the peak is in general a function of the chain length $N$ and the chain stiffness $\kappa_{b}$. Two special cases are worth considering separately as shown in Fig. 8. For a fully flexible chain, the position of the peak shifts at a higher $s$-value for longer chain (Fig. 8(a)); for a given chain length $N$, this peak shifts towards a lower $s$-value for a stiffer chain as shown in Fig. 8(b). For $\kappa_{b} \neq 0$, the position of the peak in general will depend on the ratio $t_{t p} /\langle\tau\rangle$, as will be discussed in Sec. III D. The noteworthy point from all these figures for the waiting time distribution is the fact that $W(s)$ is non-monotonic in $s$ reaching maximum for some $\tilde{s}\left(N, \kappa_{b}\right)$. This characteristic implies a time dependent friction on the monomer for a finite chain length $N$ as discussed below using TP picture.

\section{Connection with the TP theory}

We now relate this $t_{t p}$ obtained directly from the $\left\langle x_{N}(t)\right\rangle$ with the peak position of the waiting time distribution of the

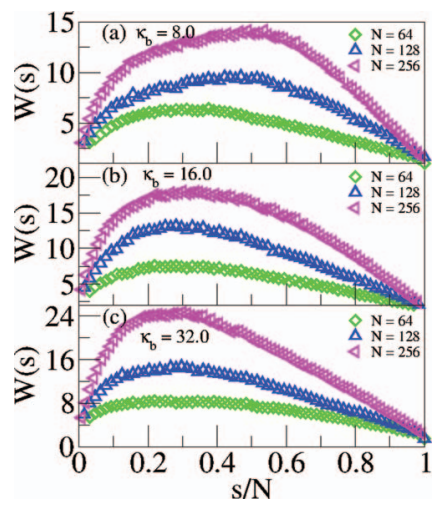

FIG. 9. Residence time of the individual monomers as a function of $\kappa_{b} \neq 0$ for chain length $N=64,128$, and 256 . The symbols have the same meaning as in Fig. 8(a).

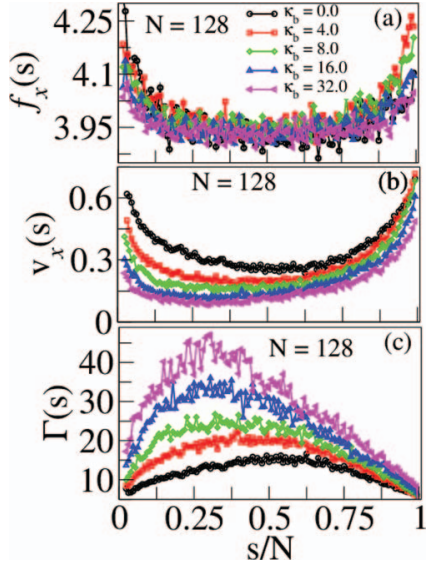

FIG. 10. The total (a) force $f_{x}(s)$ and (b) velocity $v_{x}(s)$ along the direction of translocation, and (c) the total friction $\Gamma(s)$ on the $s$ coordinate for a chain length $N=128$. A comparison of $W(s)$ and $\Gamma(s)$ shows that the waiting time distribution is the finger print of the friction experienced by the monomer inside the pore.

individual monomers. According to the recent BDTP theory proposed by us ${ }^{22,23}$ this peak corresponds to the tension propagation time $t_{t p}$. We will provide a brief physically appealing argument here. The details can be found in references. ${ }^{22,23}$ Let us denote $\tilde{s}$ so that $W_{\max }(s)=W(\tilde{s})$. Physically a peak in $W(s)$ implies that the monomer $\tilde{s}$ spends maximum amount of time inside the pore compared to the rest of the monomers. We will provide arguments below and show explicitly (Fig. 10) that this corresponds to maximum drag force experienced by the monomer $\tilde{s}$ using tension propagation picture.

The key idea of the TP theory is to divide the cis side subchain into two distinct (near and far) domains where the distances are measured from the pore. The monomers in the (near) domain closer to the pore move towards the pore being dragged by the external force. The far domain consists of immobile (on an average) monomers yet to respond to the driving force. For a finite chain of length $N$, the total time dependent viscous drag experienced by the monomer $\tilde{s}$ inside the pore $\Gamma(t)=\gamma_{c i s}(t)+\gamma_{\text {pore }}$. Since the external bias is constant, assuming a force balance ${ }^{6}$ implies a time dependent $\tilde{v}(t)=F / \Gamma(t)$. The time dependent viscous drag $\Gamma(t)$ inside the pore becomes maximum when maximum number of monomers at the cis side participate in the translocation

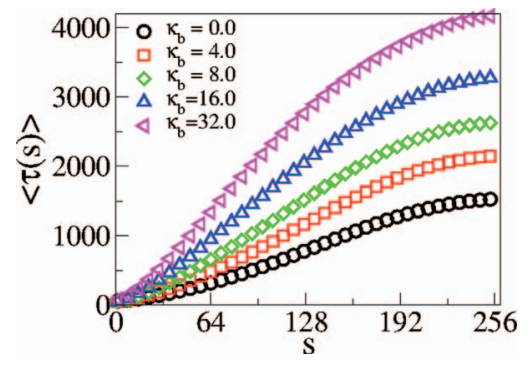

FIG. 11. MFPT $\langle\tau(s)\rangle$ for the $s$ coordinate for a $\mathrm{N}=256$ chain for different values of the stiffness parameter $\kappa_{b}$. The symbols have the same meaning as in Fig. 5. 
TABLE I. Comparison of the ratio $\frac{t_{t p}}{\langle\tau\rangle}$ for different values of $\kappa_{b}$ for chain length $N=128$ monitoring $\left\langle x_{N}(t)\right\rangle$ and using Eq. (11), respectively.

\begin{tabular}{rcc}
\hline \hline$\kappa_{b}$ & $\left(\frac{t_{t p}}{\langle\tau\rangle}\right)_{N}(t)$ & $\left(\frac{t_{t p}}{\langle\tau\rangle}\right)_{W_{\max }}$ \\
\hline 0.0 & 0.58 & 0.60 \\
4.0 & 0.53 & 0.53 \\
8.0 & 0.49 & 0.48 \\
16.0 & 0.40 & 0.40 \\
\hline \hline
\end{tabular}

process. This happens precisely at $t=t_{t p}$ when the tension front reaches the last monomer. For $t>t_{t p}$, the number of monomers start decreasing at the cis side as they translocate to the trans side and the viscous drag decreases. This explains the shape of the waiting time distribution of Figs. 8 and 9. We have further looked into this aspect of time dependent friction by monitoring the components of velocity and force along the direction of translocation as shown in Fig. 10. The ratio $\Gamma(s)=f_{x}(s) / v_{x}(s)$ is the friction experienced by the monomer inside the pore $s$ exhibits a maximum and has the same qualitative feature (Fig. 10(c)) as that of $W(s)$ confirming the friction becomes maximum at the tension propagation time $t_{t p}$.

Therefore, in order to test the TP theory, we have also measured $t_{t p}$ from position of $W_{\max }$ using Eq. (11),

$$
\sum_{s=1}^{\tilde{s}}\langle W(s)\rangle=t_{t p}
$$

(which is a direct consequence of the TP theory) as follows: (i) First, we have used the plots of $W(s)$ as a function of $s$ to identify $\tilde{s}$ which corresponds to the maximum of $W(s)$ $\left(W_{\max }=W(\tilde{s})\right)$; (ii) then we have used the simulation data for $\langle\tau(s)\rangle$ (Fig. 11 below) to obtain $\langle\tau(\tilde{s})\rangle$. During the simulation $\langle\tau(s)\rangle$ was recorded as each monomer arrived at the pore. In Table I, we show the $t_{t p}$ calculated both by the di-

TABLE II. $\tilde{s}, t_{t p},\langle\tau\rangle$, and the ratio $\frac{t_{t p}}{\langle\tau\rangle}$ for different values of $\kappa_{b}$ for chain length $N=64,128$, and 256 .

\begin{tabular}{rrrrrr}
\hline \hline$N$ & $\kappa_{b}$ & $\tilde{s}$ & \multicolumn{1}{c}{$t_{t p}$} & $\langle\tau\rangle$ & $\frac{t_{t p}}{\langle\tau\rangle}$ \\
\hline 64 & 0.0 & 26 & 85.02 & 174.80 & 0.48 \\
& 4.0 & 21 & 103.17 & 236.93 & 0.43 \\
& 8.0 & 19 & 119.28 & 290.51 & 0.41 \\
& 16.0 & 16 & 125.69 & 348.36 & 0.36 \\
& 32.0 & 15 & 140.83 & 413.19 & 0.34 \\
128 & 0.0 & 63 & 342.14 & 572.81 & 0.59 \\
& 4.0 & 54 & 390.97 & 737.31 & 0.53 \\
& 8.0 & 49 & 404.53 & 840.13 & 0.48 \\
& 16.0 & 35 & 453.67 & 1139.74 & 0.39 \\
& 32.0 & 31 & 463.67 & 1296.22 & 0.35 \\
256 & 0.0 & 142 & 904.79 & 1548.16 & 0.58 \\
& 4.0 & 126 & 1195.69 & 2188.94 & 0.54 \\
& 8.0 & 106 & 1378.90 & 2831.01 & 0.48 \\
& 16.0 & 84 & 1225.21 & 3221.25 & 0.38 \\
& 32.0 & 70 & 1425.00 & 4124.31 & 0.34 \\
\hline \hline
\end{tabular}

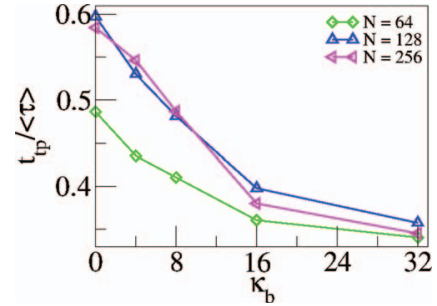

FIG. 12. $t_{t p} /\langle\tau\rangle$ for different values of $\kappa_{b}$ for chain length $N=64$ (green diamonds), 128 (blue up-triangles, and 256 (magenta left-triangles), respectively.

rect method from $\left\langle x_{N}(t)\right\rangle \sim t$ and from the waiting time distribution using Eq. (11). The excellent agreement of the $t_{t p}$ obtained by two different methods clearly establishes the validity of the nonequlibrium TP theory on a firmer ground in the context of polymer translocation problem. Since it is relatively easier to calculate $t_{t p}$ from the $W(s)$ and we have shown that both methods provide the same value $t_{t p}$, we have used Eq. (11) to determine the tension propagation time $t_{t p}$ for various chain lengths and chain stiffness (Table II). For larger chain length $N$, the ratio $t_{t p} /\langle\tau\rangle$ becomes almost independent of the chain length and decreases with chain stiffness as expected (Fig. 12).

Finally we would like to discuss how the tension propagation time varies as a function of the external bias. It is expected that the $t_{t p}$ will decrease for larger bias. In Eq. (11), we have shown the connection of $t_{t p}$ with the waiting time distribution. Previously, for fully flexible chains it has been found that $\langle\tau\rangle \sim F^{-1}$. From Eq. (10), one expects that plot of $W(s) \cdot F$ versus $s / N$ for different bias will fall onto the same master curve. Plots of $W(s)$ as a function of $s$ for different biases are shown in Fig. 13. Since $\langle\tau\rangle$ decreases as the bias increases, the $W_{\max }$ also decreases as the area under each curve is exactly equal to $\langle\tau\rangle$ (Eq. (10)). The inset of Fig. 13 shows the scaled plot $F \cdot W(s)$ as a function of $s$ which exhibits reasonably good scaling as expected. In the limit of very long chain this scaling will become exact. Likewise, Fig. 14 shows the plot of $\left\langle x_{N}(t)\right\rangle$ for different values of the external force inside the pore. The inset (a) shows the peak position of second derivative $d^{2}\left\langle x_{N}(t)\right\rangle / d t^{2}$ which clearly shows that the $t_{t p}$ decreases for larger bias. The inset (b) shows the collapse of $\left[d^{2}\left\langle x_{N}(t)\right\rangle / d t^{2}\right] \cdot\langle\tau\rangle^{2}$ when plotted as a function of $t /\langle\tau\rangle$. Since $\langle\tau\rangle \propto F^{-1}$, this collapse shows that $t_{t p} \propto F^{-1}$.

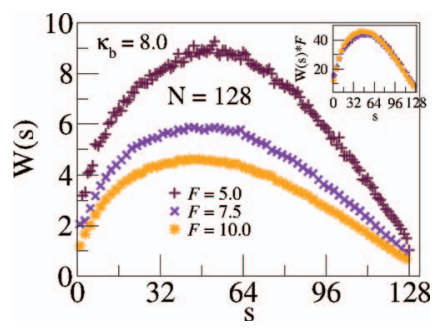

FIG. 13. Residence time for a chain $N=128$ with $\kappa_{b}=8.0$ for different biases $F=5.0$ (chocolate plus), 7.5 (violet $\mathrm{X}$ ), and 10.0 (orange stars), respectively. The inset shows the corresponding data collapse for $W(s) \cdot F$. 


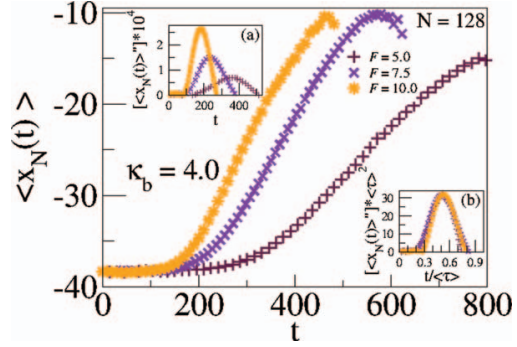

FIG. 14. $\left\langle x_{N}(t)\right\rangle$ as a function of $t$ for different values of external bias. Insets (a) and (b) show the corresponding 2nd derivatives as a function of $t$ and $t /\langle\tau\rangle$, respectively. The later shows that the ratio $t_{t p} /\langle\tau\rangle$ is almost independent of the external bias.

\section{SUMMARY AND DISCUSSION}

To summarize, we have extended the study of polymer translocation through a nanopore for a semi-flexible chain and studied how chain flexibility affects various properties of a translocating chain. First we showed that the MFPT increases for a stiffer chain and argued that this is primarily due to chain elongation. We have also observed that MFPT for different chain stiffness satisfies $\left\langle\tau\left(\kappa_{b}\right)\right\rangle \sim\left\langle\tau\left(\kappa_{b}=0\right)\right\rangle \exp$ $\left(-\epsilon_{b}\right)$, where $\epsilon_{b}$ is the bond energy corresponding to a stiffness $\kappa_{b}$ and therefore, is a function of the chain persistence length $l_{p}$. The other significant outcome of these studies is to validate the physical picture of TP using computer simulation data. The TP theory for driven polymer translocation captured the nonequlibrium aspects of driven translocation; however it was soon found that modification and extension of the TP theory for a finite chain is essential to rationalize a large set of not only simulation data but for correct interpretation of experimental data. An immediate consequence of finite chain effect is the TP time $t_{t p}$ which we have seen introduces a nonmonotonic time dependent drag force as demonstrated in BDTP simulation studies. However, the BDTP formalism does not involve a coarse-grained bead-spring chain as used in here. By directly monitoring the time dependence of the last monomer, we calculated this TP time $t_{t p}$ and validated the consequence of TP by showing that at $t_{t p}$, the waiting time distribution of the corresponding monomer is indeed maximum. We have also shown that scaling of $\langle\tau\rangle \sim F^{-1}$ implies that the ratio $t_{t p} /\langle\tau\rangle$ is independent of $F$ for a given chain length $N$ and bending constant $\kappa_{b}$.

Finally we would like to make some remarks about recent results for two-dimensional semi-flexible chains ${ }^{55}$ and show its relevance in the context of polymer translocation problem. Unlike $3 \mathrm{D},{ }^{56}$ a semi-flexible chains in $2 \mathrm{D}$ do not have a Gaussian regime because of severe dominance of excluded volume (EV) effect in $2 \mathrm{D} .{ }^{55}$ In $2 \mathrm{D}$, a chain behaves like a rod for $n=L / l_{p}<1\left(L=(N-1) b_{l}\right.$, the contour length), however for larger $L / l_{p}>1$, it crosses over to an EV chain invalidating the well known Kratky-Porod relation $R_{N}^{2}=2 l_{p} L\left\{1-\frac{1}{n}[1-\exp (-n)]\right\},{ }^{47}$ which predicts Gaussian behavior $R_{N}^{2}=2 l_{p} L$ for large $n$. However, a 3D EV semiflexible chain while crossing over from the rod to the EV chain for a limited range of $n$ behaves like a Gaussian chain. ${ }^{56}$ Therefore, in order to extract the translocation exponent for semi-flexible chains in 2D, one has to first identify if the com- binations $\left(N, l_{p}\right)$ reside in the rod limit or EV limit. Only in either of these two limits correction scaling analysis for the translocation exponents for a semi-flexible chain will be useful to study effects of pore friction leading to an anomalous scaling exponent. ${ }^{24}$ We have checked that the chain lengths and stiffness parameters considered in this paper lie in the crossover region. ${ }^{57}$ Much longer chains are needed to extract the translocation exponent properly from the correction to scaling ansatz recently proposed by us. ${ }^{24}$ These calculations are order of magnitude more extensive in computations; but when done the results can be used to interpret experimental data for polymer translocation through nanopores. ${ }^{57}$ We hope our work will lead to future work in this direction.

\section{ACKNOWLEDGMENTS}

The research has been supported in part by the NSFCHEM (Grant No. 0809821) and a seed grant from UCF College of Sciences and Office of Research and Commercialization. We thank Professor Kurt Binder and Professor Gary Slater for various discussions and the referees for constructive criticisms on the manuscript.

${ }^{1}$ M. Muthukumar, Polymer Translocation (CRC Press, Boca Raton, 2011).

${ }^{2}$ A. Milchev, J. Phys.: Condens. Matter 23, 103101 (2011).

${ }^{3}$ B. Alberts et al., Molecular Biology of the Cell (Garland Publishing, New York, 1994).

${ }^{4}$ J. J. Kasianowicz, E. Brandin, D. Branton, and D. Deamer, Proc. Natl. Acad. Sci. U.S.A. 93, 13770 (1996).

${ }^{5}$ J. L. Li, M. Gershow, D. Stein, E. Brandin, and J. A. Golovchenko, Nature Mater. 2, 611 (2003); A. J. Storm, J. H. Chen, X. S. Ling, H. W. Zandbergen, and C. Dekker, ibid. 2, 537 (2003).

${ }^{6}$ A. J. Storm, C. Storm, J. Chen, H. Zandbergen, J.-F. Joanny, and C. Dekker, Nano Lett. 5, 1193 (2005).

${ }^{7}$ A. Meller, L. Nivon, E. Brandin, J. Golovchenko, and D. Branton, Proc. Natl. Acad. Sci. U.S.A. 97, 1079 (2000); A. Meller and D. Branton, Electrophoresis 23, 2583 (2002).

${ }^{8} \mathrm{See} \mathrm{http://www.nanoporetech.com} \mathrm{for} \mathrm{nanopore} \mathrm{sequencing.}$

${ }^{9}$ R. dela Torre, J. Larkin, A. Singer, and A. Meller, Nanotechnology 23, 385308 (2012).

${ }^{10}$ M. J. Kim, M. Wanunu, D. C. Bell, and A. Meller, Adv. Mater. 18, 3149 (2006).

${ }^{11}$ W. Sung and P. J. Park, Phys. Rev. Lett. 77, 783 (1996).

${ }^{12}$ M. Muthukumar, J. Chem. Phys. 111, 10371 (1999).

${ }^{13}$ D. K. Lubensky and D. Nelson, Biophys. J. 77, 1824 (1999).

${ }^{14}$ J. Chuang, Y. Kantor, and M. Kardar, Phys. Rev. E 65, 011802 (2001).

${ }^{15}$ Y. Kantor and M. Kardar, Phys. Rev. E 69, 021806 (2004).

${ }^{16}$ J. L. A. Dubbeldam, A. Milchev, V. G. Rostiashvili, and T. Vilgis, Phys. Rev. E 76, 010801(R) (2007); Europhys. Lett. 79, 18002 (2007).

${ }^{17}$ J. K. Wolterink, G. T. Barkema, and D. Panja, Phys. Rev. Lett. 96, 208301 (2006).

${ }^{18}$ D. Panja, G. T. Barkema, and R. C. Ball, J. Phys.: Condens. Matter 19, 432202 (2007); 20, 075101 (2008).

${ }^{19}$ H. Vocks, D. Panja, G. T. Barkema, and R. C. Ball, J. Phys.: Condens. Matter 20, 095224 (2008).

${ }^{20}$ T. Sakaue, Phys. Rev. E 76, 021803 (2007); 81, 041808 (2010).

${ }^{21}$ P. Rowghanian and A. Y. Grosberg, J. Phys. Chem. B 115, 14127 (2011).

${ }^{22}$ T. Ikonen, A. Bhattacharya, T. Ala-Nissila, and W. Sung, Phys. Rev. E 85, 051803 (2012).

${ }^{23}$ T. Ikonen, A. Bhattacharya, T. Ala-Nissila, and W. Sung, J. Chem. Phys. 137, 085101 (2012).

${ }^{24}$ T. Ikonen, A. Bhattacharya, T. Ala-Nissila, and W. Sung, "Scaling theory of driven polymer translocation," Europhys. Lett. (submitted); e-print arXiv: $1211.7043 \mathrm{v} 1$.

${ }^{25}$ J. L. A. Dubbeldam, V. G. Rostiashvili, A. Milchev, and T. A. Vilgis, Phys. Rev. E 85, 041801 (2012).

${ }^{26}$ A. Milchev, K. Binder, and A. Bhattacharya, J. Chem. Phys. 121, 6042 (2004) 
${ }^{27}$ K. Luo, T. Ala-Nissila, S-C. Ying, and A. Bhattacharya, J. Chem. Phys. 126, 145101 (2007).

${ }^{28}$ K. Luo, T. Ala-Nissila, S.-C. Ying, and A. Bhattacharya, Phys. Rev. Lett. 99, 148102 (2007); 100, 058101 (2008).

${ }^{29}$ K. Luo, T. Ala-Nissila, and S.-C. Ying, J. Chem. Phys. 124, 034714 (2006); 124, 114704 (2006).

${ }^{30}$ I. Huopaniemi, K. Luo, T. Ala-Nissila, and S.-C. Ying, J. Chem. Phys. 125, 124901 (2006)

${ }^{31}$ D. Wei, W. Yang, X. Jin, and Q. Liao, J. Chem. Phys. 126, 204901 (2007).

${ }^{32}$ S. Matysiak, A. Montesi, M. Pasquali, A. Kolomeisky, and C. Clementi, Phys. Rev. Lett. 96, 118103 (2006).

${ }^{33}$ S. Guillouzic and G. W. Slater, Phys. Lett. A 359, 261 (2006).

${ }^{34}$ M. G. Gauthier, and G. W. Slater, J. Chem. Phys. 128, 065103 (2008); 128, 205103 (2008); Eur. Phys. J. E 25, 17 (2008); M. G. Gauthier, and G. W. Slater, Phys. Rev. E 79, 021802 (2009).

${ }^{35}$ M. Kenward and G. W. Slater, Eur. Phys. J. E 20, 125 (2006); Phys. Rev. E 78, 051806 (2008).

${ }^{36}$ H. W. de Haan and G. W. Slater, J. Chem. Phys. 134, 154905 (2011); 136, 154903 (2012).

${ }^{37}$ K. Luo, S. Ollila, I. Huopaniemi, T. Ala-Nissila, P. Pomorski, M. Karttunen, S.-C. Ying, and A. Bhattacharya, Phys. Rev. E 78, 050901(R) (2008).

${ }^{38}$ K. Luo, T. Ala-Nissila, S.-C. Ying, and R. Metzler, Europhys. Lett. 88, 68006 (2009).

${ }^{39}$ R. Metzler and K. Luo, Eur. Phys. J. Spec. Top. 189, 119 (2010).

${ }^{40}$ A. Bhattacharya, W. H. Morrison, K. Luo, T. Ala-Nissila, S.-C. Ying, A. Milchev, and K. Binder, Eur. Phys. J. E 29, $423-429$ (2009).
${ }^{41}$ A. Bhattacharya and K. Binder, Phys. Rev. E 81, 041804 (2010).

${ }^{42}$ V. V. Lehtola, R. P. Linna, and K. Kaski, Europhys. Lett. 85, 58006 (2009); Phys. Rev. E 78, 061803 (2008).

${ }^{43}$ V. V. Lehtola, K. Kaski, and R. P. Linna, Phys. Rev. E 82, 031908 (2010).

${ }^{44}$ A. Bhattacharya, Phys. Procedia 3, 1411 (2010).

${ }^{45} \mathrm{~A}$. Bhattacharya, "Translocation dynamics of a semi-flexible chain under a bias: Comparison with tension propagation theory" J. Polym. Sci. (to be published).

${ }^{46}$ J. Comer and A. Aksimentiev, J. Phys. Chem. C 116, 3376 (2012); Nanoscale 2, 468 (2010).

${ }^{47}$ M. Rubinstein and Ralph H. Colby, Polymer Physics (Oxford University Press, 2003).

${ }^{48}$ A. Yu. Grosberg and A. R. Khokhlov, Statistical Physics of Macromolecules (AIP Press, 1994).

${ }^{49}$ P. G. de Gennes, Scaling Concepts in Polymer Physics (Cornell University Press, Ithaca, 1979).

${ }^{50}$ G. S. Grest and K. Kremer, Phys. Rev. A 33, 3628 (1986).

${ }^{51}$ H.-P. Hsu, and K. Binder, J. Chem. Phys. 136, 024901 (2012).

${ }^{52}$ D. W. Schaefer, J. F. Joanny, and P. Pincus, Macromolecules 13, 1280 (1980).

${ }^{53}$ H. Nakanishi, J. Phys. 48, 979 (1987).

${ }^{54}$ W. F. van Gunsteren and H. J. C. Berendsen, Mol. Phys. 45, 637 (1982).

${ }^{55}$ H.-P. Hsu, W. Paul, and K. Binder, Eur. Phys. Lett. 95, 68004 (2011).

${ }^{56}$ H.-P. Hsu, W. Paul, and K. Binder, Eur. Phys. Lett. 92, 28003 (2010).

${ }^{57}$ A. Huang, R. Adhikari, A. Bhattacharya, and K. Binder (unpublished). 